\title{
La reforma del sector salud, descentralización, prevención y control de enfermedades transmitidas por vectores
}

\author{
Health system, decentralization, \\ and the control of vector-borne diseases
}

Gabriel A. Schmunis 1

João Carlos Pinto Dias 2

\footnotetext{
1 Programa de Enfermedades Transmisibles, Organización Panamericana de la Salud. 525 Twenty-third Street, N.W. Washington DC, 20037, USA. schmunig@paho.org

2 Centro de Pesquisas René Rachou, Fundação Oswaldo Cruz. C.P. 1743, Belo Horizonte, $M G$ 30190-002, Brasil. jcpdias@cpqrr.fiocruz.br
}

\begin{abstract}
Economic policies are changing Latin American health programs, particularly promoting decentralization. Numerous difficulties thus arise for the control of endemic diseases, since such activities traditionally depend on vertical, and centralized structures. Theoretical arguments in favor of decentralization notwithstanding, no such tradition exists at the county level. The lack of program expertise at peripheral levels, intensive staff turnover, and even corruption are additional difficulties. Hence, the simple bureaucratic transfer of activities from the Federal to county level is often irresponsible. The loss of priority for control of endemic diseases in Latin America may mean the inexorable extinction of traditional control services. Malaria, dengue fever, and Chagas disease programs are examples of the loss of expertise and effectiveness in Latin America. A better strategy for responsible decentralization is required. In particular, a shared transition involving all governmental levels is desirable to effectively modernize programs. Maintenance of regional reference centers to ensure supervision, surveillance, and training is suggested.
\end{abstract}

Key words Prevention and Control; Health Care Reform; Chagas Disease

Resumen Con las nuevas macropoliticas mundiales, la salud en América Latina ha sufrido importante transición en direción a la decentralización, sin compatibilizar la salud pública con la lógica de las economías de mercado. Con esto, el control decentralizado de las enfermedades endémicas presenta dificultades políticas y operativas. Aunque la decentralización se justifica por los presupuestos teóricos, no hay tradición de este control en los niveles municipales, lo que dificulta la simple o burocrática transferencia de encargos para estos niveles. La falta de expertise, el turn-over político y la corrupción son dificultades adicionales, conllevando a una extinción de varias instituiciones y programas. La falta de efectividad en el enfrentamiento del dengue, de la malaria y de la enfermedad de Chagas son algunos ejemplos. Requierese una modernización con responsabilidad, con una transición compartida entre los niveles y garantizada por acciones continuadas. Sugierese mantener estructuras regionales para referencia, consolidación epidemiológica, normatización, capacitación y supervisión, incluso con reserva técnica para acciones finalísticas supletivas.

Palabras clave Prevención y Control; Reforma Sanitária; Doença de Chagas 


\section{El contexto}

La presente década encuentra a los paises latinoamericanos en un periodo de crecimiento económico limitado, pero significativamente mayor que lo ocurrido en los años 80 en que las dificultades financieras dieron lugar a un ajuste macroeconómico con una disminución significativa del gasto público y social.

El proceso de democratización iniciado en la década pasada está en consolidación. El fortalecimiento de este proceso, que acompana a las agendas políticas, sociales y económicas de la mayoria de los países, ocurre en un contexto de globalización económica cuya justificación es la disminución de costos. La respuesta de defensa de los países ha sido la integración regional o subregional, como mecanismo destinado a mejorar la productividad y la competitividad en los mercados internacionales. La intensificación de las tendencias privatistas y la disminucion de las burocracias estatales trajeron como consecuencia a una pérdida del personal capacitado (y no capacitado), cuyo empleador era el Estado, y como subproducto un aumento de la desocupación (Sojo, 1997; Ugalde \& Jackson, 1997; Bossert, 1998).

A lo anterior se suma un cambio en el rol del Estado en búsqueda de eficiencia, efectividad, y sustentabilidad que, se pregona, faltan en las instituciones públicas. En el sector público en salud, a esta justificación se le adiciono el aumento en el costo de las prestaciones, lo que requiere una mayor racionalidad en el uso de los recursos disponibles, cada vez mas escasos en relación al costo incrementado de prestaciones médicas más complejas (OPS, 1997a; Sojo, 1997; Ugalde \& Jackson, 1997; Bossert, 1998).

Sin embargo, existe un nuevo énfasis discursivo en la revalorización de la agenda social destinada a superar las inequidades en la cobertura y en el acceso a los servicios entre los distintos estratos sociales. Inequidades que han aumentado como consecuencia de la globalización de las economías y la disminución del gasto público con destino social (Londoño \& Frenck, 1997; Dias, 1998; H. Málaga, comunicación personal).

En el sector salud, esto coincide con un cambio en el rol del sector público, caracterizado por la disminución en la provisión de servicios que se transfieren a los niveles periféricos (Provinciales, Estaduales o Municipales) y com el fortalecimiento en el desarrollo de políticas, en el liderazgo normativo y en su función reguladora, tanto a través de la provisión de financiamiento como de servicios. Esta redefinición de los roles se da por medio de la reorga- nización de los sistemas de salud a través de la descentralización y de la redefinición de los modelos de atención (OPS, 1997a; Sojo,1997; Ugalde \& Jackson, 1997; Bossert, 1998).

En teoria, de acuerdo a perfiles epidemiológicos locales (patologías prevalentes), es posible definir paquetes de prevención y atención prioritarios, en función de los recursos disponibles. La gestión de estos últimos se haría, a su vez, más eficiente, cuando los proveedores públicos compiten con los privados. Por otra parte, los hospitales, origen de gran parte del gasto en salud, se reorganizarían empresarialmente, bridandoles autonomía de gestión y oportunidad de recuperación de costos. Asi, la existencia de diversos prestadores facilitaría la competencia entre los mismos y estimularia la capacidad de selección del (de los) usuario(s) y del (de los) financiador(es). De esta manera, se estimularía la competencia entre el sector público y el privado, y se podrían ejecutar reformas de gestión a menudo centradas en una evaluación periódica del desempeño.

Una característica comun a casi todos los procesos de reforma fue el énfasis en las campañas sanitarias, en la atencion primaria, en las intervenciones focales en grupos de riesgo y en la instauración de prestaciones básicas universales, subsidiando la demanda, la atención ambulatoria y el autocuidado (Sojo, 1997).

Un proceso de estas características requiere la participación de numerosos actores, su adecuada interacción y el desarrollo de la capacidad de negociación para la adquisición de consensos que permitan una reforma que cumpla con las aspiraciones de cada una de las partes intervinientes. La meta final es prevenir y/o evitar las desigualdades innecesarias, evitables e injustas en las condiciones de salud de la población que afectan a los más pobres, grupos de riesgo con enfermedades específicas o que poseen barreras geográficas de acceso a los servicios. Básicamente, la reforma está destinada a paliar el efecto de la falta de cobertura de los servicios, tanto en lo que se refiere a población cubierta como a brindar prestaciones efectivas (Sojo, 1997).

No es de extrañar que, en América Latina (y en otros continentes), las mayores desigualdades en salud afectan a los más pobres. Asi, la carga de enfermedad del $40 \%$ de éstos es cuatro veces superior a la que afecta al $20 \%$ más rico. Por otra parte, el $40 \%$ de la población de ingresos medios posee una carga de enfermedad que es la mitad de la de los pobres, pero, todavia, es el doble de la de los ricos. En este marco, los procesos de reforma tendrían como objetivo fundamental el tratar de alcanzar la equidad 
(Dias, 1995; Londoño \& Frenck, 1997; Sojo, 1997; H. Málaga, comunicación personal).

Si bien es temprano en el tiempo como para juzgar definitivamente si se alcanzan el objetivo fundamental de la reforma del sector salud, la experiencia en lo que cabe a la atención hospitalaria muestra que, durante la ejecución del proceso de reforma, disminuyó la demanda de los estratos más pobres de la población (Laguna, 1999).

La presente comunicación tiene por objeto analizar el impacto del proceso de descentralización en la región, en relación a los programas de control de vectores, tratando de señalar pros y cons del mismo y sus posibles consecuencias.

\section{Los programas tradicionales de control de vectores y la situación de las enfermedades transmitidas por vectores}

A continuación, se resume la situación en relación a las enfermedades transmitidas por vectores: dengue, malaria y enfermedad de Chagas, que, por su prevalencia e incidencia, producen un daño significativo en la población y un impacto económico negativo de magnitud en los países endémicos.

Históricamente, estos programas se caracterizaron por su estructura vertical, semi-militar, con una rígida organización; personal muy disciplinado e imbuido de su misión; logística con abundante dotación de material y equipo; y recursos humanos y financieros suficientes. Aunque por lo habitual dependian del Ministerio de Salud, en la práctica se manejaban como entes autónomos. El personal no discutía las ordenes, no había sindicato que peleara por mejores condiciones de trabajo y el uniforme de mata-mosquitos se vestía con orgullo.

La cobertura para combatir al enemigo, el vector de turno, era total. Su expresión más acabada fueron los programas de erradicación de la malaria y del Aedes aegypti y de prevención de la transmisión vectorial del Trypanosoma cruzi.

\section{Dengue y fiebre amarilla urbana}

El control de la fiebre amarilla durante la construcción del canal de Panamá fue un éxito en la década del 20. Ésto fue el resultado del esfuerzo de miles de hombres ( $10 \%$ de la población de la Ciudad de Panamá estaba destinada al control de la Fiebre Amarilla), para reducir las fuentes de mosquitos con recursos que, aunque en la actualidad parecen primitivos, fueron muy efectivos. Un éxito similar se obtuvo en Rio de Janeiro, Brasil, en esa misma década.

El uso intensivo del DDT, sumado, en ocasiones, a la reducción de criaderos, hizo del $A$. aegypti una especie casi erradicada de la región en la mitad de la década de los sesenta. Sólo quedaban focos en los EUA, en Venezuela, y en los países del Caribe. Si bien este éxito fue transitorio, ya que el A. aegypti volvió a reinfestar a los países y actualmente sólo Chile y Canadá estan libres del vector, el fracaso surgió de la falta de percepción de los gobiernos, que olvidaron que la prevención de reinfestaciones dependía no sólo de la instalación de la vigilancia entomológica, sino que también de la ejecución de acciones larvicidas y adulticidas como respuesta al hallazgo del mosquito. La consecuencia fue una constante disminución de los recursos nacionales dedicados a la solución del problema, tanto en personal como en diñero (OPS, 1997b).

Aunque hasta ahora ese fracaso fue sólo parcial, ya que, desde 1942, desapareció la fiebre amarilla urbana de las Américas, la situación del dengue y de la fiebre hemorrágica del dengue (FHD) se agravó, aumentando progresivamente el número de casos en la región, año tras año (OPS, 1997b; Schmunis \& Corber, en prensa).

La previsión de que acciones coordinadas entre países serían capaces de re-erradicar el vector se tornaron poco realistas, cuando el costo de estas acciones se estimó en 1.600 millones de dólares anuales, durante tres o cuatro anos y, posteriormente, en una suma substancial de recursos para mantener la vigilancia y evitar las reinfestaciones, para que no se repitiera la historia de la erradicación previa. Si todo programa requiere para su éxito de factibilidad técnica, viabilidad económica, y aceptabilidad social, el programa de erradicación del $A$. aegypti, aunque técnicamente factible, resultó política, económica y socialmente inviable (OPS, 1997c).

\section{Malaria}

A partir de la década de los 40, las campañas de control del mosquito vector con DDT y, en ocasiones, obras de ingeniería erradicaron la malaria donde las condiciones sociales y ecológicas asi lo permitían. Expresión de ese éxito fue la erradicación del Anopheles gambiae, especie exótica importada de África al Brasil, la erradicación de la malaria urbana en gran parte de los países donde existia, y la importante disminución del area geográfica rural donde existía transmisión. 
Las campañas de erradicación tenían una objetivo claro y medible, erradicar la malaria o el Aedes. El tiempo necesario para ejecutar las acciones y terminar con el problema era finito. Sin embargo, la falta de condiciones para alcanzar el objetivo de erradicación de la malaria hizo que la campaña se interrumpiera, cuando se comprobó que las condiciones sociales y ecológicas hacían imposible el éxito. Además, la falta de un tiempo límite para conseguir el éxito descorazonó a los países proveedores de fondos.

La malaria aun hoy continua siendo endémica en 23 países de la región donde se produjeron 1.200.000 casos en 1998. El area de Plasmodium falciparum se encuentra en la región Amazónica de Bolivia, Brasil, Ecuador, Guyana, Guyana Francesa, y Surinam, donde ocurrieron decenas de miles de casos de malaria producidos por esa especie. Más del 50\% de todos los casos de malaria ocurrieron en esos países. En Centro América y en México, donde el Plasmodium vivax es endémico; ocurrieron 182.000 casos de malaria en 1998 (OPS, 1998).

Aunque el número de casos de malaria aumentó en los países amazónicos, con la excepción de Brasil, en las areas con P. falciparum, se detectó una disminución significativa en la mortalidad por malaria. De 890 defunciones en 1990 a 260 en 1994. Este suceso se debió al aumento en el número de servicios que disponen de drogas antimaláricas; desde cloroquina, hasta drogas como la piridoxina/sulfadiazina, artemisinina, quinina y/o mefloquina, para las cuales el $P$. falciparum todavía es suceptible (OPS, 1998; Gusmão, 1999). Sin embargo, el incremento observado en la morbilidad sugiere que acciones de prevención y control dirigidas contra el vector todavía tendrán su indicación en anos futuros.

\section{Enfermedad de Chagas}

En los países del Cono Sur, los programas verticales eliminaron el vector de gran parte de Argentina y Brasil. En Chile, las acciones fueron ejecutadas por las regiones, bajo la coordinación del Ministerio de Salud, que proveyó de los fondos. En Uruguay, las acciones las llevaron a cabo los departamentos y las municipalidades, con la cooperación técnica y logística del nivel central. En todos los casos, los recursos fueron suministrados casi por completo por el Estado. La excepción fue el Uruguay en que, por un año, los recursos fueron suministrados por las municipalidades.

En los países del Cono Sur, la transmisión vectorial del T. cruzi por Triatoma infestans a sido interrumpida en Uruguay, en 1997, y en
Chile, en 1999 (WHO, 1997; TDR, 1999). Asimismo, el area de distribución del T. infestans a disminuido significativamente en Argentina y Brasil, y existen progresos en el control vectorial en Bolivia y Paraguay. Si bien el T. infestans es el principal vector del T. cruzi en los países del Cono Sur, y se espera su eliminación debido a sus caracteristícas exclusivamente domiciliarias (Schmunis et al., 1997), el control de otras especies selváticas que invaden la vivienda, tales como Triatoma brasiliensis, Triatoma sordida, o Pastrongilus megistus, indica que las acciones de rociado para el control de esas especies de triatomineos seran todavía una necesidad, aunque se elimine el T. infestans. Lo mismo ocurriría en el norte de Sud América, Colombia y Venezuela, donde el principal vector es el Rhodnius prolixus, especie que, en esa area geográfica, es considerada selvática que invade la vivienda (Schofield, 1994).

En Centro América, aun cuando se elimine el $R$. prolixus, especie considerada intradomiciliaria en esta subregión, la existencia de Triatoma dimidiata, especie selvática que invade la vivienda y que se encuentra aun en la perifería de ciudades capitales, como Tegucigalpa y Managua, sugiere que las acciones de control deberan tener una duración indefinida (OPS, 1999).

\section{Situación actual de los programas}

En general, los recursos financieros son menores a los existentes previamente. La única excepción es Brasil, en que la campaña contra el Aedes requiere de ingentes recursos que, aunque provistos por el nivel central, se ejecutan a través de las municipalidades.

La provisión de fondos del nivel central a los municipios, como bolsa comun, sin especificar destino y asi definir su uso específico, hizo que los mismos, en ocasiones, se usen en actividades diferentes de las originalmente planificadas. Sin embargo, esto aun ocurrió cuando a los recursos se les dió un destino específico. En Brasil, por ejemplo, la proliferación de municipios coincidió con una disminución del gasto en salud, en educación y en servicios públicos (The Economist, 1998; Fonseca, 1999).

La profusión de recursos humanos técnicamente capacitados que caracterizaba los programas tradicionales, ese ejercito de técnicos en la lucha contra los vectores, está en vías de extinción o ya está extinto. En Centro América, poco queda del personal experto de los programas de control de malaria y Aedes. Los existentes han sido distribuidos en los servicios, don- 
de su actividad se diluye. En Argentina y Brasil, la disminución del personal operativo para el control de triatomineos a sido significativa. En el primero, de casi mil funcionarios a principios de la década de los 90, sólo quedan la mitad. En el segundo, de los 9.000 funcionarios existentes en la mitad de la década pasada, se han reducido a 2.300 en la actualidad. Con el agravante que el deterioro de la situación del dengue (OPS, 1997c) requiere cada vez más la cooperación de ese personal técnico en el control del Aedes en situaciones de emergencia.

En muchos de los países el personal experto del nivel central o fue eliminado de la plantilla de personal del programa, como consecuencia del ajuste y de la reorganización del sector, o fue transferido a unidades ejecutoras provinciales, departamentales o municipales en areas de la descentralización, dependiendo de cual fuese la estructura orgánica del país en cuestión. Una excepción que vale mencionar es Paraguay, en el cual la estructura del Servicio Nacional de Erradicación de la Malaria (SENEPA) todavía se mantiene. En Goiás, Brasil, la Fundación Nacional de Salud ha tomado la decisión de decentralizar el programa de control de dengue, ahora compartido con las municipalidades, y mantener verticalizado el control de Chagas hasta que se elimine el principal vector regional, el T. infestans. Con esto, prácticamente previnió el riesgo de perder en la transición operativa, de programa dependiente del nivel central a programa municipal, los logros ya alcanzados com el sistema tradicional bien organizado.

En otros países, la transferencia de personal hizo que, en muchos casos, su experticia original desapareciera, ya que son utilizados en funciones diferentes para la que fueron preparados cuando se desempeñaban en el programa institucional nacional. Este personal, al ser transferido a unidades ejecutoras, se diluye en numerosas unidades de atención, donde no se reconoce su conocimiento y experiencia y se los ocupa en funciones no relacionadas con la misma.

La experiencia comun es que las provincias, departamentos, o municipios tuvieron dificultades para responder al reto del control de vectores en forma completa, aunque, en teoría, posean los recursos. Ya sea porque los recursos son desviados para otros fines; porque carecen de interés, ya que que el control de vectores no es considerado prioritario; o porque carecen de los recursos humanos necesarios en cantidad y calidad, o éstos estan diluidos entre el resto del personal, o ya no existen, y/o el programa del nivel central posee una disminuida capacidad de respuesta, que compense por las fallas de la acción en el nivel de descentralización elegido por el país. En particular, y mirando el panorama futuro del control de las grandes endemías en Latinoamérica, el horizonte indica una prioridad teórica para las actividades de vigilancia epidemiológica, que no vienen siendo contempladas de manera sactisfactoria en la práctica operativa por los países, estados o municípios. Esto es un desafío urgente para todos los sectores involucrados, que también demanda una íntima y permanente cooperación de las comunidades con los sectores de gobierno (Dias, 1998).

A lo anterior, y como elemento negativo dentro del proceso de descentralización actual en el control de los vectores, se suma la existencia de recursos financieros atomizados, logística limitada, y capacidad de compra restringida. Esto ocurre tanto por los menores recursos disponibles, como por ser menor la cantidad necesaria de insumos, debido a la disminución del area geográfica, ámbito de acción del programa. En consecuencia, al disminuir la economía de escala, aumentan los costos.

Un elemento que complica aun más la situación futura de los programas de control son las tendencias actuales de "globalización", "tercerización” y "privatización”. La filosofía prevalente en la economía mundial, basada en la economía de mercado, no garantiza una prioridad para la atención de las poblaciones más pobres, quienes son justamente las más afectadas por esas enfermedades. Esto conlleva a la necesidad de una formulación de políticas de salud y de inversión social, en principio se contradice con un simple achicamento del Estado. En este sentido, ponencias de la comunidad científica, como de la Sociedad Brasileña de Medicina Tropical, aclaran que, aunque sea la decentralización un camiño justo y deseable en el perfeccionamiento de los sistemas de salud, la simple desactivación burocrática de programas y estructuras actuantes, como la Fundación Nacional de Salud de Brasil, no constituye actos políticos responsables, si los municipios o estados no tienen como absorber las actividades de control pertinentes. Esto es aun más contundente para el caso de los municipios más pobres de las regiones más deprimidas socialmente.

Como parte de un proceso saludable de municipalización, habría que pensar en un proceso gradual y progresivo de capacitación local, con supervisión y coordinación regional, que aproveche al máximo los recursos humanos disponibles en los programas nacionales, (Dias, 1993, 1995). Por otro lado, la ventaja de la 
descentralización fue obvia cuando la accion de control se realizó por medio de los servicios generales de salud. Éste fue el origen del suceso que significó la baja de la mortalidad como consecuencia del mejor acceso de la población en riesgo a servicios de salud, con la capacidad de brindar diagnóstico y tratamiento de malaria (Gusmão, 1999).

\section{El futuro en el contexto de la realidad actual}

La situación actual, aun considerando la dificultad política del renacimiento de los programas institucionales autónomos, dirigidos del nivel central, requiere el repensar en que situaciones la dilución de esfuerzos que caracteriza la descentralización en el control de los vectores origina peligros de salud pública que no justifican el riesgo de mantener una descentralización a ultranza, con la desaparición de los programas hoy existentes.

En el futuro, el horizonte operativo estará indudablemente basado en el fortalecimiento de la vigilancia epidemiológica (Dias, 1993; Meelgard et al., 1998). Esto presupone una acción permanente, con respuestas rápidas y diferenciadas por cada situación (Dias, 1998).

Una característica vital de los programas tradicionales fueron la cobertura total y el hecho que las acciones tenian una duracion limitada en el tiempo. En el primer elemento, la propia ejecución del proceso de descentralización dificulta este tipo de acciones. Imaginemos un programa de control del dengue en un país como Brasil, con más de 5.000 municipios. La posibilidad de coordinar a todos ellos en acciones de erradicación (o control) será dificil de mantener en el tiempo, aun cuando los fondos sean provistos por el nivel central. Un sólo municipio que no lleve a cabo las acciones, con la prontitud y efectividad requerida, será fuente de infestación de los otros municipios que lo circundan, obligandolos a intensificar sus acciones contra el mosquito y disminuyendo los recursos disponibles para otros fines.

El segundo elemento, la existencia de acciones limitadas en el tiempo, descarta que acciones de control se puedan llevar a cabo ahi donde las condiciones sociales y/o ecológicas no aseguren el éxito en un tiempo definido. La imposibilidad de cumplir con ambas premisas descarta, en el caso de la malaria y del dengue, la implementación de programas de erradicación tanto a cargo del nivel central como descentralizados. Incluso en Brasil, con sus ingentes recursos destinados al control del A. aegyp- $t i$, la falta de acción en los países vecinos, hace que las medidas de vigilancia y control antivectorial deberían ser mantenidas a lo largo y ancho del territorio por tiempo indefinido, debido al intenso grado de infestación de los países vecinos.

Los programas nacionales requieren de personal capacitado y movil, capaz de responder con soltura a las emergencias de malaria y dengue a nivel mas periférico y que, al mismo tiempo, sean capaces de capacitar al personal a ese nivel. De tal manera que el proceso de descentralización constituya una descentralización responsable; por lo menos en lo que se refiere a la disponibilidad de personal capacitado para analizar la situación local, reaccionar frente a esta situación y solicitar la ayuda del programa nacional si la situación lo amerita. Pero ese personal del nivel central debe existir. Esto mismo es válido para la ejecución del control de especies de triatomineos no domiciliarios.

Por el contrario, la eliminación del T. infestans, éxito pasible de ser alcanzado si se ejecutan acciones de cobertura nacional y duración definida, ya aceptadas por lo países, todavía justifica la existencia de un programa institucional nacional responsable por el control vectorial. La falta de utilización o no existencia de un programa con estas características conspira para que no se alcancen las metas regionales de eliminación de triatomineos domiciliarios sobre los que el rociado con piretroides de acción residual es probadamente efectivo. 


\section{Referencias}

BOSSERT, T., 1998. Analyzing the decentralization of health systems in developing countries: Decision space, innovation and performance. Social Science and Medicine, 47:1513-1527.

DIAS, J. C. P., 1993. O papel do município no controle da doença de Chagas no Brasil e a proposta de extinção da Fundação Nacional de Saúde. Revista da Sociedade Brasileira de Medicina Tropical, 26 (Sup. 2):47-50.

DIAS, J. C. P., 1995. Aspectos políticos em doença de Chagas. Revista da Sociedade Brasileira de Medicina Tropical, 28 (Sup. 3):41-45.

DIAS, J. C. P., 1998. Problemas e possibilidades de participação comunitária no controle das grandes endemias no Brasil. Cadernos de Saúde Pública, 14 (Sup. 2):19-37.

FONSECA, A., 1999. Prefeitos desviam dinheiro da Educação. A Tarde, Salvador, 24 mai., Notícias Regionais, pp. 1, 3, 13 .

GUSMÃO, R., 1999. Overview of Malaria control in the Americas. Parasitologia, 41:1-6.

LAGUNA, J. A., 1999. La autonomización de hospitales públicos y sus consecuencias sobre la demanda de atención de salud, Lima, 1988-1997. Revista de Salud Pública, 6:301-310.

LONDOÑO, J. L. \& FRENCK, J., 1997. Structured Pluralism: Towards a New Model for Health System Reform in Latin America. Washington, D. C.: Pan American Health Organization/World Health Organization. (mimeo.)

MELGAARD, B.; CREESE, A.; AYLWARD, B.; OLIVÉ, J. M.; MAHER, C.; OKWO-BELE, J. M. \& LEE, J. W., 1998. Disease eradication and health systems development. Bulletin of the World Health Organization, 76 (Sup. 2):26-31.

OPS (Organización Panamericana de la Salud), 1997a. La Descentralización, los Sistemas de Salud y los Procesos de Reforma del Sector. Informe Final. Serie 1: Organización y Gestión de Sistemas y Servicios de Salud. Valdivia: OPS.

OPS (Organización Panamericana de la Salud), 1997b. Resurgimiento del dengue en las Américas. Boletín Epidemiológico (OPS), 18:1-7.
OPS (Organización Panamericana de la Salud), 1997c. Plan Continental de Ampliación e Intensificación del Combate al Aedes aegypti. Informe OPS/ HCP/HCT/90/97. Caracas: OPS.

OPS (Organización Panamericana de la Salud), 1998. Situación de los Programas de Malaria en las Américas, 1997. XLVI Informe OPS/HCP/HCT/ M/17. Washington, D.C.: OPS.

OPS (Organización Panamericana de la Salud), 1999. Primera Reunión de la Comisión Intergubernamental de la Iniciativa Centroamericana y Belice para la Interrupción de la Transmisión Vectorial de la Enfermedad de Chagas por Rhodnius prolixus, disminución de la Infestación Domiciliaria por Triatoma dimidiata, y Eliminación de la Transmisión Transfusional del Trypanosoma cruzi. Documento OPS/HCP/HCT/145/99. Washington, D.C.: OPS.

SCHMUNIS, G. A.; ZICKER, F. \& MONCAYO, A., 1997. Interruption of Chagas' disease Transmission through vector elimination. Lancet, 398:11711174.

SCHMUNIS, G. A. \& CORBER, S. Tourism and emerging and reemerging infectious diseases in the Americas. What physicians must remember for patient diagnosis and care. Brazilian Journal of Infectious Diseases. (en prensa)

SCHOFIELD, C. J., 1994. Triatominae. Biology and Control. London: Eurocomunica Publications.

SOJO, A., 1997. La reforma finisecular del sector salud en América Latina y el Caribe. In: Las Políticas de Salud en el Umbral de la Reforma (United Nations Children's Emergency Fund - UNICEF, ed.), Serie de Políticas Sociales No. 1, pp. 48-54, San José: UNICEF.

TDR (Tropical Diseases Research), 1999. Chile and Brazil to be certified free of transmission of Chagas disease. TDR News, 59:10.

THE ECONOMIST, 1998. Brazil local loot. The Economist, Washington, D.C., May 15, p. 38.

UGALDE, A. \& JACKSON, J. T., 1997. Las políticas de salud del Banco Mundial: Una revisión crítica. Cuadernos Médico-Sociales, 73:45-60.

WHO (World Health Organization), 1997. Chagas disease. Interruption of transmission. Weekly Epidemiological Record, 72:1-5. 